\title{
Impulsive Breakdown Characteristics of Solid-Gas Interfaces
}

\author{
Z. Wang, Graduate Student Member, IEEE, I. Timoshkin, Senior Member, IEEE, M. P. Wilson, Member, IEEE, \\ M. J. Given, Senior Member, IEEE, T. Wang, S. J. MacGregor, Senior Member, IEEE
}

\begin{abstract}
Comprehensive knowledge and understanding of the breakdown behavior of solid-gas interfaces is important for the development, optimisation and coordination of insulation in practical high voltage gas-insulated systems (GIS), which are widely used in the power and pulsed power industries. However, despite significant research efforts focused on characterisation of the breakdown parameters and properties of complex gas-filled HV systems, the basic mechanisms which underpin the development of discharges in such solid-gas topologies are not fully understood. This lack of knowledge is particularly noticeable in the case of solid-gas interfaces stressed with high voltage impulses. The present study is aimed at the experimental investigation of the breakdown behavior of solid-gas interfaces, formed between solid dielectrics and common gases: dry air, $\mathrm{N}_{2}$ and $\mathrm{CO}_{2}$, at atmospheric pressure. Four different solid dielectric materials, PTFE, HDPE, Delrin and Macor, with relative permittivities in the range from 2.1 to 6.3, were used in the present work. The solid-gas interfaces formed by these dielectric materials and the surrounding gases were stressed with positive and negative HV impulses. The breakdown voltage and time to breakdown were obtained for all combinations of solid-gas interfaces using both, positive and negative, impulses, with a nominal rise time of $\sim 1.9 \mu$ s. A digital camera operated in the open shutter regime was used to obtain images of the breakdown spark channels, which were classified according to their propagation paths. The results obtained in the present study will help in further understanding of the breakdown behavior of solid-gas interfaces, and can be used in the development and optimization of dielectric topologies for use in gas-insulated systems.
\end{abstract}

Index terms - impulsive breakdown voltage, time to breakdown, solid-gas interface, spark breakdown path.

\section{INTRODUCTION}

$\mathrm{S}_{\mathrm{s}}^{\mathrm{o}}$ lid dielectric spacers are commonly used in high voltage, gas-insulated systems to provide mechanical support to high voltage (HV) electrodes. There are a number of critically important parameters that should be considered in the design of HV, power and pulsed power, gas-insulated systems (GIS). These parameters include not only the bulk breakdown properties of insulating gases and solid dielectrics, but also the surface flashover characteristics, i.e. the breakdown (flashover) voltages associated with the initiation and propagation of discharges across solid-gas interfaces. The surface flashover properties of solid-gas interfaces are important for insulation coordination in HV, gas-filled systems; understanding of, and detailed information on, the dielectric behavior of such interfaces supports the design and development of compact, $\mathrm{HV}$ and pulsed power topologies and components, such as gasinsulated transmission lines and gas-filled plasma closing switches.

Another critical aspect that should be addressed when designing $\mathrm{HV}$ and pulsed power systems, is the use of environmentally friendly gases. The employment of such gases in GIS and other gas-insulated HV components and systems is aimed at potential replacement of $\mathrm{SF}_{6}$ - an insulating gas with excellent dielectric and switching characteristics, but with a significant global warming potential (GWP), 23,900 times that of $\mathrm{CO}_{2}$. A systematic and comprehensive investigation of the characteristics and parameters of the flashover events across solid-gas interfaces in environmentally friendly gases, and in gases with low (as compared with $\mathrm{SF}_{6}$ ) GWP values, is required for further understanding, optimisation and coordination of dielectric insulation in different practical, gas-filled, HV power and pulsed power systems and their elements, [1], [2].

It is known that both, DC and impulsive, flashover voltages across solid-gas interfaces can be lower than the breakdown voltage of the same electrode topologies filled only with gas, with no solid dielectric spacers bridging the inter-electrode gap, [3], [4]. One of the most problematic points in gas-filled HV systems, which requires special attention from the point of view of preventing breakdown, are so-called triple points/junctions, i.e. points of contact between a solid dielectric and a metallic electrode in a gas atmosphere. Such triple points result in the re-distribution and enhancement of the electrical field in their vicinity, which may lead to the occurrence of partial discharges, and to a complete flashover/breakdown event in the system, [5].

However, despite a number of studies, the breakdown behavior of such interfaces under pulsed power conditions is not fully understood. Potential dependencies of the flashover/breakdown voltage and time to breakdown on gas type, relative permittivity of the solid spacer material, polarity of the applied HV impulses, and other factors are not well known, and require further investigation.

There are multiple parameters which may affect the breakdown behavior of solid-gas interfaces. For example, in [2] the effect of the relative permittivity of solid insulators on the breakdown strength of different solid-gas interfaces was discussed. It was argued that dielectric spacers with lower permittivity induce a lower field enhancement at triple points, while the material with higher relative permittivity increases the electric field in this region resulting in a decrease in breakdown strength. In [6], the breakdown behavior of two electrode topologies with solid dielectric sheets, termed "perpendicularfield" and "parallel-field" topologies, were investigated in ambient air. In the case of the "parallel-field" topology, a solid dielectric sheet was located vertically, perpendicular to the horizontally-located plane grounded electrode, while the pin HV electrode was positioned at the top of the solid dielectric sheet. In this geometry, there was a strong tangential (parallel to the surface of the solid dielectric sheet) electric field component. It was found that, within the range of relative permittivities of solid dielectric materials used in the study, 
from $\varepsilon_{r}=2.1$ to $\varepsilon_{r}=3.9$, the flashover voltage across solid sheet-air interfaces in the "perpendicular-field" topology did not depend upon $\varepsilon_{r}$. However, in the case of the "perpendicularfield" topology, when an additional grounded metallic plate was located at the opposite side of the dielectric sheet (perpendicular to the grounded plane electrode), a decrease in the breakdown voltage was observed with an increase in the $\varepsilon_{r}$ of the dielectric sheet. In this topology, there is a strong perpendicular (normal to the solid dielectric sheet) electric field component.

In [3], the DC flashover voltage was obtained for three different types of solid insulator, Lucite, Textolite, and Isolantite, in a $\mathrm{N}_{2}$ environment. It was found that the corrugated surface of these solid insulators led to an increased flashover voltage as compared with a smooth surface, and that this difference in the flashover voltages becomes greater with an increase in the gas pressure. The highest DC flashover voltage was reported for the spacers made of Lucite, [3].

However, the published data on the breakdown/flashover behavior of solid-gas interfaces do not provide a complete picture required for a comprehensive analysis of the breakdown mechanisms and characteristics of such interfaces. For example, while the spark channel length and its location are important factors required for comprehensive characterisation of flashover events, there is insufficient information on the path of the breakdown channel(s) formed in solid-gas insulation systems. The spark channel length is a factor which, among others, defines the impedance of the breakdown spark channel, and the intensity of the pressure impulse/wave generated by this spark discharge; such high-pressure impulses can cause substantial damage to solid insulators in the HV system. Thus, these factors and characteristics are important for optimisation and insulation coordination and should be taken into account in the design of practical, gas-filled, HV power and pulsed power systems and components.

The path of the breakdown channel is governed by the prebreakdown streamer(s) propagating through the gas-solid insulation systems. The initiation mechanisms of streamers, their propagation characteristics and parameters have been the subject of a number of experimental and analytical studies. In [6], it was found that, in highly-divergent electric fields, there were two streamer branches which initiate simultaneously from a single HV point electrode, and propagate simultaneously through the bulk of air and along the solid-air interface. At 50\% breakdown voltage level, only the streamer which develops through the bulk of the air was able to bridge the inter-electrode gap, transforming into a breakdown spark channel. However, incomplete streamer(s) may introduce substantial space or surface charge into the system. These residual charges may affect the development of subsequent streamer(s), if the insulation system is stressed with multiple HV impulses. Understanding of the residual charge effects is important for characterisation of the breakdown properties of solid-gas insulation systems under repetitive impulsive energisation.

A study of the flashover voltage across different solid-gas interfaces conducted in ambient air, [6], also demonstrated that an increase in the magnitude of the applied positive HV impulses leads to a change in the path of the breakdown spark channel. In [6], three different spark channel paths were described to characterise the channel location: breakdown through the bulk of the gas, complete flashover across the solidgas interface, and partial flashover across the solid-gas interface.

In [7], breakdown spark channels and their location in insulation systems formed by electrodes partially embedded into a Lexan dielectric plate, in both air and $\mathrm{N}_{2}$, were studied. The electrode system used in this study provided a non-uniform but symmetrical electric field between the anode and cathode. It was shown that in the case of a $\mathrm{N}_{2}$ atmosphere, breakdown channels detached from the solid-gas interface and propagated above the Lexan surface, through the bulk of the $\mathrm{N}_{2}$, following the electric field lines. However, in the case of air, the breakdown channels developed across the Lexan-air interface. The authors argued that, in the case of electronegative gas(es) (air in this case), oxygen molecules attach free electrons, and the attachment process impedes the development of spark channels through the bulk of the gas; while in the case of an electropositive gas, $\mathrm{N}_{2}$, the ionization wave(s) propagates through the bulk of the gas, resulting in the formation of the breakdown channel along the (bended) field lines above the solid dielectric. This study indicates that the gas properties may significantly affect the path of the spark breakdown channel in solid-gas insulation systems.

Multiple physical processes, such as: the emission of secondary electrons from the dielectric surface due to UV photons generated by the propagating ionisation fronts, [8], the formation of residual surface charge and the development of space charge, can also influence the spark channel development. It was shown in [9], [10], and [11] that, during propagation of the ionisation front in $\mathrm{N}_{2}$, the energy of photons produced by collisions of free electrons with neutral $\mathrm{N}_{2}$ molecules is higher than the work functions of some solid dielectrics, such as polyethylene. Thus, depending on their polarity, the ionisation fronts in $\mathrm{N}_{2}$ can stimulate secondary electron emission from solid dielectric materials, leading to the formation of positive surface charge. It was argued in [12] that, in some cases, such positive surface charge can promote the interfacial development of the spark channel. However, the role of the surface charge in the interfacial breakdown process is still not fully understood, and requires further investigation.

The present study is focused on experimental investigation of the flashover/breakdown behavior of the solid-gas interfaces formed between samples of different solid materials and gases at atmospheric pressure. The gases which have been selected for this study are $\mathrm{CO}_{2}, \mathrm{~N}_{2}$ and dry air; the solid dielectric spacers used in the breakdown tests were made from the commonly-used engineering plastics, PTFE, HDPE and Delrin (POM), and also from the machinable ceramic Macor.

An analysis of the breakdown behavior of the interfaces formed by the chosen solid materials and gases has been conducted, with the breakdown voltage and time to breakdown obtained for lightning impulses of both polarities, in a nonuniform electric field. All breakdown tests were conducted in the over-stressed energisation regime: all breakdown events occurred either on the rising slope of each $\mathrm{HV}$ impulse, or at the 
peak of the applied voltage waveform, i.e. each individual applied HV impulse resulted in complete breakdown. Open shutter photographs of breakdown spark channel(s) were obtained for each breakdown event. A systematic analysis of the breakdown paths and their correlation with gas type, solid material, and impulse polarity was conducted in this paper.

\section{EXPERIMENTAL SETUP}

\section{A. Test cell setup}

In order to investigate the breakdown behavior of the solidgas interfaces, a sealed test cell, equipped with an upper high voltage electrode and a lower grounded electrode, was designed and manufactured. The body of the test cell was made of a Perspex tube, with an internal diameter of $185 \mathrm{~mm}$ and an external diameter of $190 \mathrm{~mm}$. Two PVC discs (flanges), used to seal the test cell, were bolted to 6 outer aluminum rods, thus the flanges were firmly attached to the cylindrical body of the test cell. Rubber O-rings were used to seal the test cell.

The upper high voltage electrode was made of a stainlesssteel rod, with a diameter equal to the diameter of solid dielectric rod samples, $10 \mathrm{~mm}$ nominally. Since there are small variations in the nominal diameters of the commercially obtained cylindrical solid samples, the HV electrodes were manufatured for each specific solid dielectric material sample, to match its practical diameter. The lower grounded disk electrode, with rounded edges, was also made of stainless steel, and mirror polished. To ensure that each solid dielectric sample was aligned with the top cylindrical HV electrode, $7 \mathrm{~mm}$ deep holes were drilled in the center of all dielectric samples, and a pin located at the center of the upper HV electrode was inserted into this hole. The solid cylindrical samples were located on the surface of the polished grounded electrode. Thus, each solid dielectric sample was secured and located in a known position, providing consistency in the breakdown tests.

Fig. 1 shows the test cell assembly, with a solid cylindrical dielectric sample placed between the electrodes.

The solid dielectric samples were manufactured from commercially available cylindrical rods of PTFE, HDPE, Delrin and Macor, with each rod having a nominal diameter of $10 \mathrm{~mm}$. The length of each cylindrical dielectric sample was $43 \mathrm{~mm}$, thus the length of solid-gas interface from the edge of the upper $\mathrm{HV}$ electrode to the ground electrode was $43 \mathrm{~mm}$.

The triple junction was formed around the circumference of the HV electrode where the edges of the solid dielectric sample and the electrode meet. This triple junction promoted the initiation of streamers and the formation of the breakdown spark channels. The geometry of the practical topology, including triple junctions formed between the HV electrode and cylindrical solid dielectrics, is discussed and analysed in Section E.

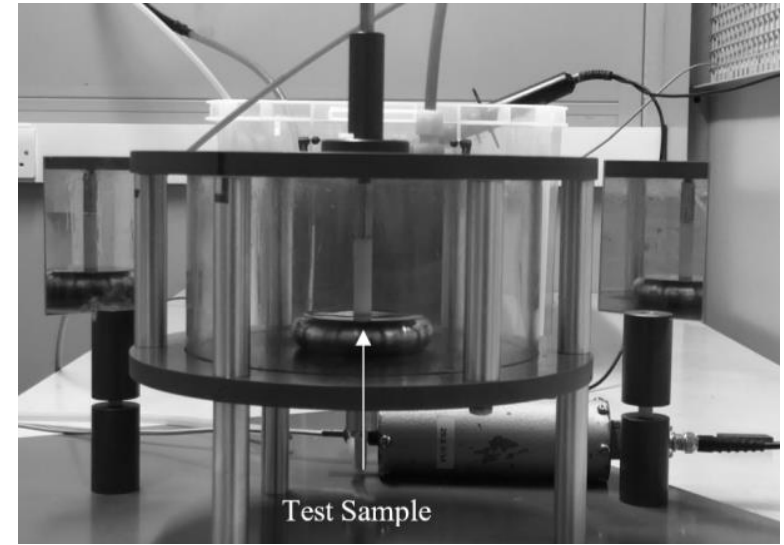

Fig. 1. The sealed test cell with a dielectric test sample located between the upper HV electrode and lower grounded electrode.

\section{B. Optical diagnostic arrangements}

To visualise and study the spark breakdown path, an open shutter photography method was used. The test set-up was equipped with a Sony RX10II camera. Optical images obtained using a direct visual path of the camera lens may not allow for an accurate identification of the breakdown channel path in the test cell. To overcome this problem, an optical system which consists of 2 mirrors located outside the test cell was used to obtain an image of all spark breakdown channels.

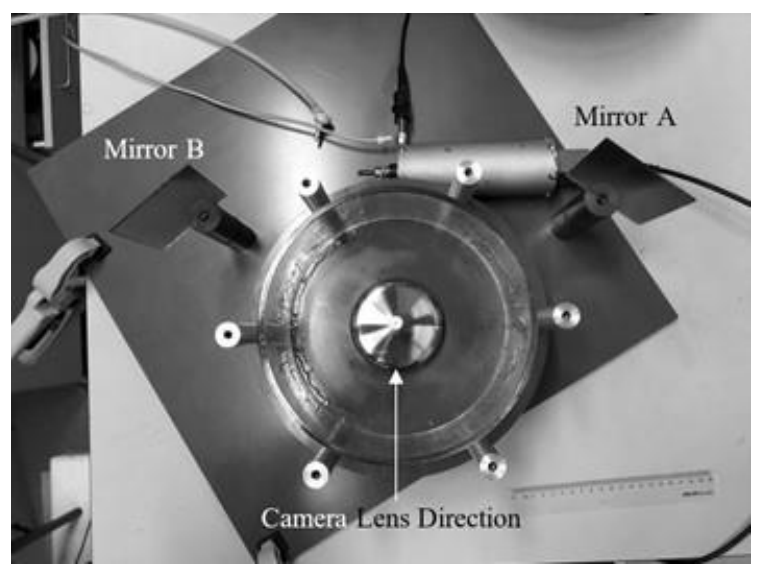

Fig. 2. Open top view on the test cell with two mirrors outside of the cell.

Fig. 2 shows a top view of the open test cell with a dielectric sample in the center, and 2 mirrors located outside of the test cell. The direct optical path of the camera lens, and the two optical paths between the mirrors and the sample, form $120^{\circ}$ angles. The mirror centers are set at the same height as the camera lens. This optical arrangement allows the camera to record optical images from three different points of view equally spaced on an arc of 360, This approach allows the identification of the location of the spark channels at any point on the surface of the insulator.

\section{HV impulse generator and diagnostics}

A four-stage, air-insulated, Marx generator was used to generate lightning impulses of both polarities. The generator was pneumatically triggered by reducing the air pressure in its 
spark column by means of a vacuum pump.

The magnitude of the DC charging voltage used to charge the capacitor bank of the Marx generator was set to $27 \mathrm{kV}$ in the case of negative HV impulses. This value was selected in order to achieve all breakdown events on the "rising" (in terms of the magnitude) slope of each negative HV impulse, or at the peak of the voltage waveform.

In the case of positive $\mathrm{HV}$ impulses, the magnitude of the DC charging voltage was set to $20 \mathrm{kV}$. The reason for selecting this lower DC charging voltage for positive energisation is as follows. It is known that, in the case of electronegative gases, positive breakdown voltages are lower than negative. The use of a higher charging voltage in the case of positive energisation led to a short time to breakdown, which was difficult to resolve due to a substantial noise component during the early stage of the voltage waveforms. This resulted in a higher degree of uncertainty in the measurements of both, the positive breakdown voltage and time to breakdown. To increase the time to breakdown in order to achieve better accuracy in measurements in the case of positive energisation, the charging voltage was lower than in the case of negative energisation.

The positive voltage waveforms were measured using a NorthStar PVM-5 HV probe (1000:1 division ratio, $80 \mathrm{MHz}$ nominal bandwidth). The peak negative voltage exceeded the maximum voltage that could be measured by the PVM-5 probe. Therefore, in the case of negative energisation, the voltage waveforms were obtained using a custom built $\mathrm{CuSO}_{4}$-water based HV divider, combined with a commercial Tektronix P6015A HV probe (1000:1 division ratio, $75 \mathrm{MHz}$ bandwidth). The output voltage signals from the NorthStar and Tektronix probes were recorded by a Tektronix TDS5034 digitising oscilloscope ( $350 \mathrm{MHz}$ bandwidth, $5 \mathrm{GS} / \mathrm{s}$ sampling rate).

The Marx generator produced HV impulses with double exponential waveforms, which have a full rise time from zero to the peak voltage of $\sim 1.9 \mu \mathrm{s}$ and a time to half-value of $\sim 50 \mu \mathrm{s}$ across a high impedance resistive load, were no breakdown occurs. In the present work, breakdown events always occurred on the "rising" slope (in terms of the voltage magnitude) or at the peak of the voltage waveform. Each breakdown event leads to a rapid, sub- $\mu$ s voltage collapse, examples of the positive and negative breakdown wave-forms are given in Section III.

\section{Gases and solid materials}

The flashover tests were conducted in atmospheres of 3 different gases, dry bottled "Zero grade" air, $\mathrm{N}_{2}$, and $\mathrm{CO}_{2}$. All gases were purchased from BOC Ltd. "Zero grade" air contains (19.9 - 21.9) $\%$ of $\mathrm{O}_{2}$, with the remaining balance of $\mathrm{N}_{2}$, and also contains the following impurities: $5 \mathrm{ppm}$ of moisture, $5 \mathrm{ppm}$ of total hydrocarbons, and $3 \mathrm{ppm}$ of $\mathrm{CO}_{2}$. The oxygen-free $\mathrm{N}_{2}$ gas used had $99.998 \%$ purity and conforms to BS 4366 . The purity of the $\mathrm{CO}_{2}$ used was $99.8 \%$, and this gas conforms to BS 4105 .

The three polymers (PTFE, HDPE and Delrin (POM)), and the machineable ceramic (Macor), selected to manufacture the dielectric spacers provided a range of relative permittivity of the spacers from $\varepsilon_{r}=2.1$ for PTFE to $\varepsilon_{r}=6.1$ for Macor.

A specific cleaning procedure was used to treat all solid samples before each breakdown test. Firstly, all cylindrical spacers made of the same material were washed in soapy water to remove any residual engineering oil or coolant introduced by the manufacturing process. The samples were then treated in an ultrasonic water bath for 15 minutes, before being washed with distilled water to remove any residuals. In the final step of the procedure, the samples were dried in a furnace at $50{ }^{\circ} \mathrm{C}$ for 5 hours. After this cleaning procedure, the samples were stored in the laboratory environment.

\section{E. Triple junction topology}

As discussed in Section I, the triple junction(s) formed between the metallic HV electrode and the solid dielectric spacer in the gas environment can promote non-complete, partial discharges, and may lead to the formation of complete spark breakdown between the HV and ground electrodes. In order to study the breakdown behaviour of the solid-gas interfaces, the electrostatic field distribution in the test system, including the field in the vicinity of the triple junction, was modelled and analysed.

Fig. 3 shows a cross section of the model solid-gas insulation system, developed in QuickField electrostatic software. This electrostatic finite element solver was used to model the Laplacian electric field, with the axisymmetrical rotational topology of the test system employed. The obtained electric field distribution is shown in Fig. 3.

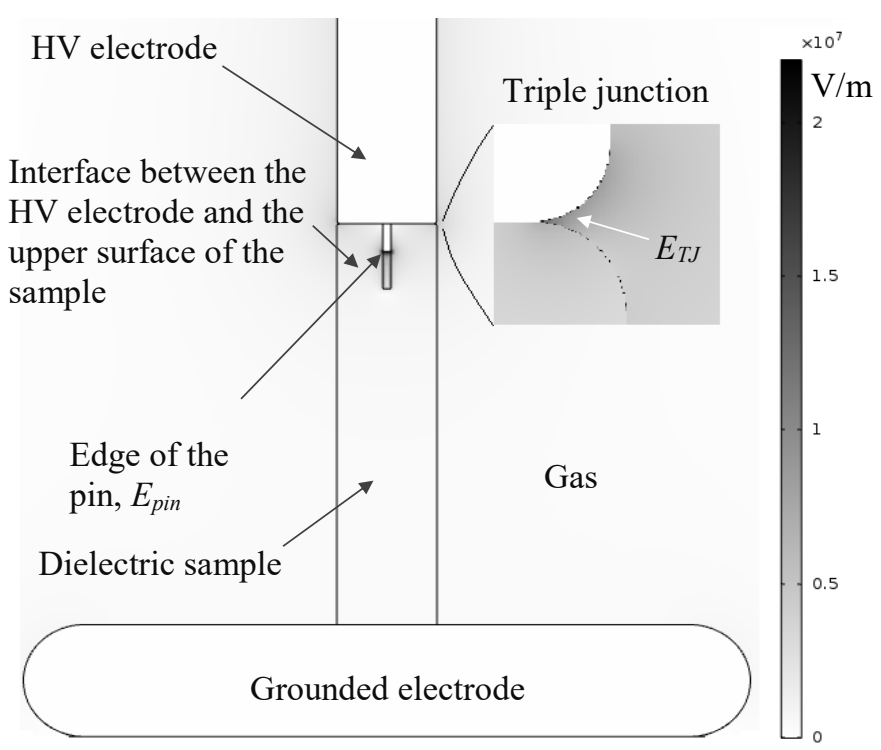

Fig. 3. Electric field magnitude in the test system. The applied voltage is $50 \mathrm{kV}$.

An exploded view of the field distribution in the vicinity of the pratical triple junction formed by the upper HV electrode and the solid spacer is also shown in Fig.3. Both the HV electrode and solid dielectric sample were modelled as having a rounded edge shown in the insert in Fig.3. This external edge is a limit in manufacturing of the samples and the electrodes. In the example shown in Fig.3, the external edge radius is $\sim 249 \mu \mathrm{m}$, and assuming that the HV potential is $50 \mathrm{kV}$, the maximum electric field at the external edge (the field at the triple junction, $E_{T J}$ ) is $\sim 200 \mathrm{kV} / \mathrm{cm}$. This electrostatic model 
also demonstrates, that the field magnitude at the edge of the pin inserted into the hole drilled in the center of the dielectric sample, $E_{p i n}$, is almost 3 times higher than $E_{T J}$. This strong electric field obviously leads to the ionization processes inside this gas-filled cavity. However, such pre-breakdown ionisation processes in the confined space within the cavity do not affect the main breakdown characteristics of the tested gas/solid interfaces, as it will be discussed in Section IV. It should be noted that the QuickField model used in the present work provides only the nominal magnitude of the Laplacian electrostatic field, no space charge which may significantly modify the electric field at the edge of the HV electrode was considered in the present model. Nevertheless, this electrostatic model provides an evaluation of the Laplacian field distribution in the test system and shows that the field at the triple junction, $E_{T J}$, is 2 order of magnitude higher than the field at the middle of gas/solid interface.

The rounded edges of the HV electrode and solid samples were modelled based on the images of the solid cylindical samples and the HV electrode obtained under an optical microscope. Fig. 4 shows a photograph of the connection between the solid spacer and the upper electrode, which was used to obtain radii of curvature of, both, the HV electrode and the solid dielectric. It was shown that, in this particular case, the edge radius of the dielectric spacer is $\sim 269 \mu \mathrm{m}$, while the radius of the edge of the HV electrode is $\sim 249 \mu \mathrm{m}$. These radii were used in the QuickField electrostatic model shown in Fig. 3.

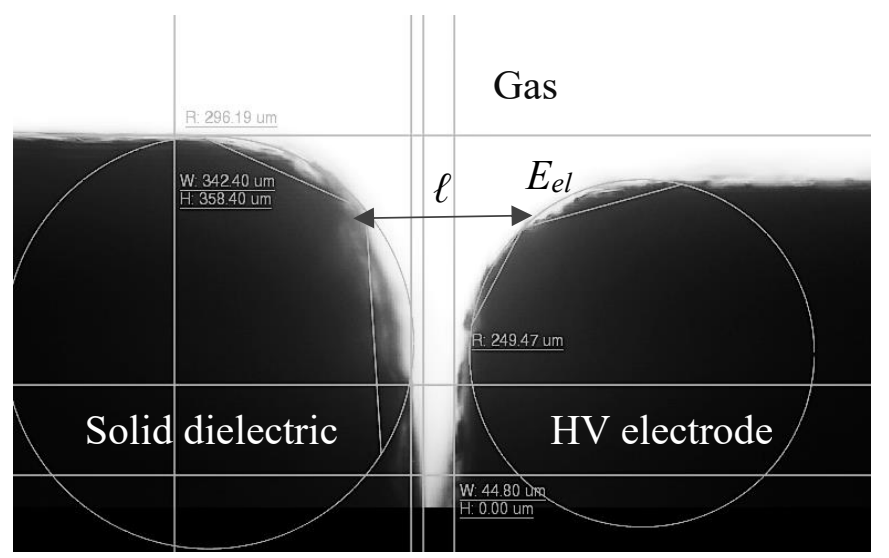

Fig. 4. A picture of the edges of a PTFE sample and HV electrode, taken using an optical microscope with mangnification of $\times 40$. Circles with radii of $\sim 249$ $\mu \mathrm{m}$ and $\sim 269 \mu \mathrm{m}$ are shown, and $\ell$ indicates the contour drawn between the HV electrode and the solid dielectric.

All solid cylindrical samples were examined under the microscope and their radii of curvature together with the radii of curvature of the corresponding $\mathrm{HV}$ electrodes are given in Table I.

TABLE I

EDGE RADII OF DIELECTRIC SPACERS AND HV ELECTRODES

\begin{tabular}{cccc}
\hline \hline Material & $\begin{array}{c}\text { Radius of edge of } \\
\text { electrode }(\mu \mathrm{m})\end{array}$ & $\begin{array}{c}\text { Radius of edge of } \\
\text { spacers }(\mu \mathrm{m})\end{array}$ & $\begin{array}{c}\text { Relative } \\
\text { permittivity } \\
\text { of spacer, } \varepsilon_{r}\end{array}$ \\
\hline
\end{tabular}

\begin{tabular}{cccc} 
Gas only & $82.0 \pm 10.0$ & $\mathrm{n} / \mathrm{a}$ & 1.0 (gas) \\
PTFE & $250.7 \pm 16.0$ & $317.9 \pm 69.9$ & 2.1 \\
HDPE & $170.8 \pm 8.84$ & $219.8 \pm 59.9$ & 2.3 \\
Delrin & $148.0 \pm 19.8$ & $725.0 \pm 194.3$ & 3.7 \\
Macor & $159.0 \pm 1.0$ & $97.0 \pm 1.0$ & 6.1 \\
$\begin{array}{l}\text { Note: } 3 \text { electrodes and } 9 \text { solid samples were used in PTFE, HDPE and Delrin tests.1 electrode and 1 } \\
\text { sample were used in Macor tests. }\end{array}$ \\
\hline
\end{tabular}

The magnitude of the electric field at the surface of the HV electrode, $E_{e l}$, depends on the distance between the HV electrode surface and the surface of the solid dielectric, $\ell$. In a cylindrical coordinate system $\ell$ is parralel to the axis of the system and its value increases as the radial position is increased. Fig 4 shows an example of the measurement of $\ell$ with the axis of the system rotated by $90^{\circ}$ to lie horizontaly.

$E_{e l}$ was obtained using the QuickField model for all 4 dieletric materials used in the present study. The highest field magnitude $E_{e l}$ at $\ell=1 \mu \mathrm{m}$ was obtained for Macor, the material with the highest relative permittivity. The normalised field magnitude, $\widetilde{E}_{e l}$ was obtained for all dielectric materails by dividing their $E_{e l}(\ell)$ values by this maximum field magnitude obtained in the case of Macor at $\ell=1 \mu \mathrm{m}$.

The normalised magnitude of the electric field at the surface of the HV electrode, $\widetilde{E}_{e l}$, obtained using the QuickField model for all dieletric materials is shown in Fig. 5 as a function of the distance between the HV electrode surface and the surface of the solid dielectric, $\ell$. It can be seen that the field magnitude on the surface of the HV electrode reduces with an increase in the distance between the electrode and the sample surface, $\ell$. However, the rate of this decrease in $\tilde{E}_{e l}$ is different for different materials. It was shown that $\widetilde{E}_{e l}$ is highest for Macor at the shortest distances, $\ell<3 \mu \mathrm{m}\left(\widetilde{E}_{e l}=1\right.$ for Macor at $\ell=1 \mu \mathrm{m}$ ); however $\tilde{E}_{e l}$ for Macor reduces fast with an increase in $\ell$ and becomes smallest amongst all tested materials when $\ell>8 \mu \mathrm{m}$. It can be seen that $\widetilde{E}_{e l}$ values are similar for all materails at $\ell \approx(5-6) \mu \mathrm{m}$.

The QuickField model provides the Laplacial electrostatic field only, the actual electric field in the vicinity of the HV elevtrode could be different due to the space charge developed during the pre-breakdown phase. The accurate modelling of the electric field during this phase which involves intensive ionisation process(es) and the development of a transient plasma front(s) is not considered in this paper. However, the electrostatic model employed in the present study can be used in the analysis of the relative field magnitudes, $\tilde{E}_{e l}$, obtained for the solid dielectrc materials with different relative permittivities. Therefore, the results shown in Fig. 5 considered within the limitations of the current electrostatic model can be used for the relative comparison of the field magnitudes at the triple junction in the case of different solid dielectrics (in the same gas and for the same polarity of the applied impulses), and in the qualitative analysis of the breakdown behaviour of the solid-gas interfaces. 


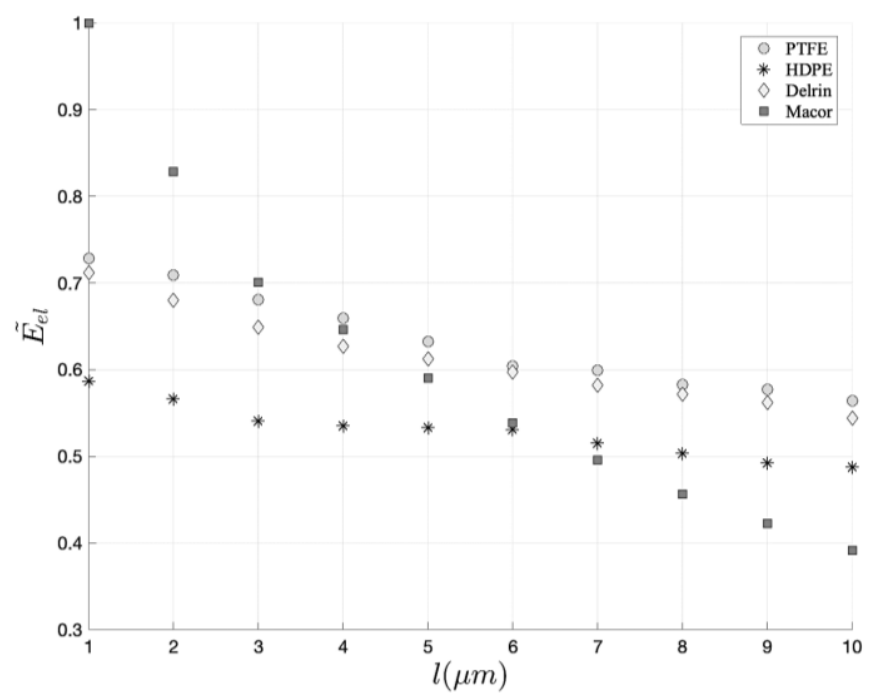

Fig. 5. Normalised electric field on surface of the HV electrode, $\widetilde{E}_{e l}$, as a function of $\ell$ for the different solid materials used in the present study.

It is known that the breakdown voltage in short, $\sim 5 \mu \mathrm{m}$ and shorter, air-filled inter-electrode gaps at atmospheric pressure deviates from the classic Paschen breakdown charateristic. The breakdown voltage in such short gaps does not increase with a decrease in the inter-electrode distance (which results in a decrease of the Paschen scaling parameter, gas pressure $\times$ gap distance). This is due to the ballistic nature of electrons in such short gaps, when their mean free path becomes comparable with the inter-electrode distance.

Thus, it could be expected that, in the present topology, the fast ionization waves (streamers) will be initiated at such locations on the surface of the HV electrode (Fig. 4), for which $\ell$ will be equal to or greater than $\sim 5 \mu \mathrm{m}$. In this case, a higher value of the relative permittivity of the solid dielectric will not necessarily result in the strongest field magnitude, $\widetilde{E}_{e l}$ on the electrode surface in the regions where streamer initiation is possible. Thus, the functional behaviour of the breakdown voltage may not demonstate an explicit dependency on the relative perimittivity. The breakdown voltage for all tested dielectric spacers and gases will be presented and discussed in Section IV.

\section{EXPERIMENTAL RESULTS}

\section{A. Voltage waveforms}

Lightning impulses of both polarities were applied to the upper HV electrode. The voltage waveforms were recorded for each breakdown event, and then used to obtain the breakdown voltage and time to breakdown.

Fig. 6 shows two example voltage waveforms, with the breakdown voltage, $V_{b r}$, and time to breakdown, $t_{b r}$, indicated. These waveforms were obtained for a flashover event across a Delrin sample in a $\mathrm{N}_{2}$ atmosphere, for negative energization.
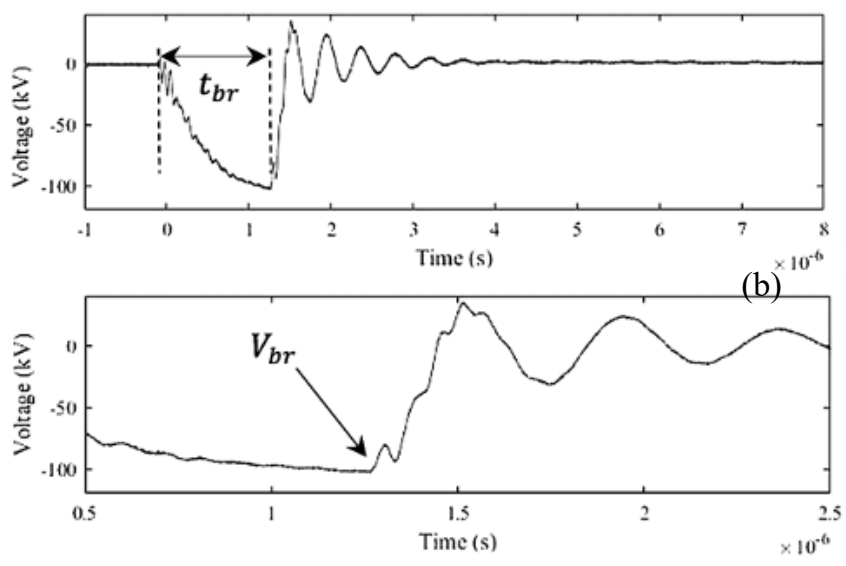

Fig. 6. Negative voltage waveform for breakdown across Delrin $/ \mathrm{N}_{2}$ interface. (a), Full wave-form; (b), zoom in to the point of breakdown.

Fig. 7 shows an example positive voltage waveform, captured in the case of flashover across a Delrin sample in a $\mathrm{N}_{2}$ atmosphere. In this case, due to the shorter, sub- $\mu$ s, time to breakdown, the recorded waveform has a noticeable noise component, and the breakdown voltage was obtained as the average value of the voltage oscillations prior to the point of breakdown (voltage collapse).
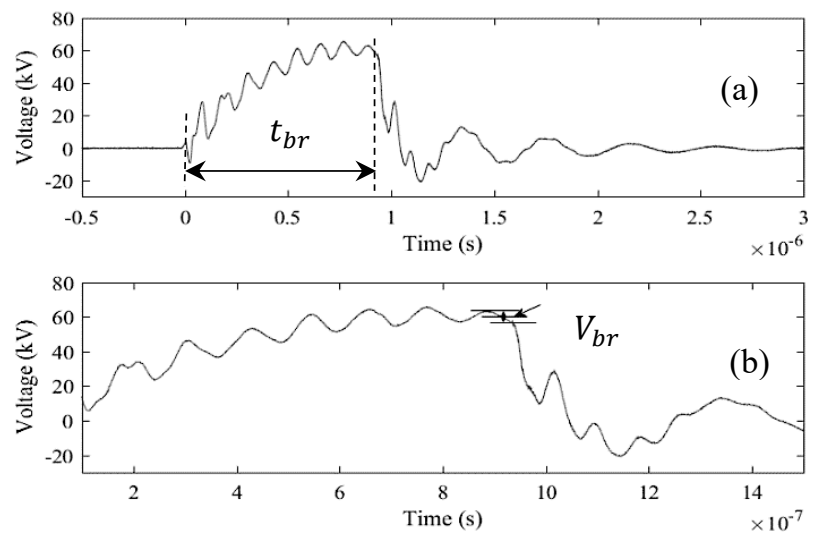

Fig. 7. Positive voltage waveform for breakdown across Delrin/ $\mathrm{N}_{2}$ interface. (a), Full waveform; (b), zoom in to the point of breakdown.

\section{B. Images of spark channels}

Open shutter images were obtained for each breakdown event. The following camera setting, 320 IOS sensitivity, and F11 aperture, and $8 \mathrm{~s}$ exposure time were used to obtain clear images of the bright post-breakdown spark channels. Prebreakdown streamer(s) were not captured due to their low brightness. The camera was triggered a few seconds prior to triggering the Marx generator.

An example of the images of a spark breakdown channel captured for one breakdown event are shown in Fig. 8: (a) frontal image taken along the main optical path between the sample and the camera lens; (b) image captured from the left mirror ' $A$ '; (c) image captured from the right mirror 'B'. 


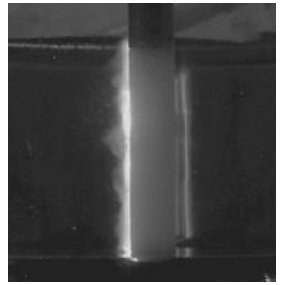

(a) Front view

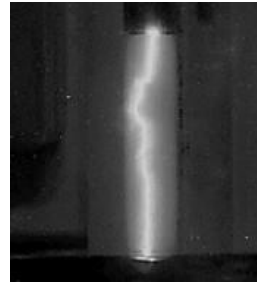

(b) Left mirror view

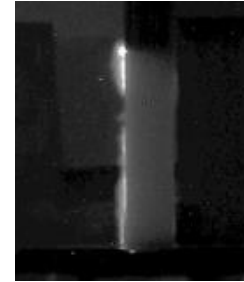

(c) Right mirror view
Fig. 8. Open shutter pictures of breakdown channels across a Delrin $/ \mathrm{N}_{2}$ interface (positive HV impulses). (a) Direct optical path; (b) left mirror image, (c) right mirror image.

It can be seen that the frontal view in Fig. 8a does not show the complete spark channel. The image obtained from the left mirror, 'A', allows for visualisation of the channel trajectory and its geometry. The image obtained from the right mirror, 'B', confirms that the channel propagated across the solid-gas interface, rather than through the bulk of the gas. This spark channel can, therefore, be classified as a complete surface flashover. The complete classification and analysis of the spark channels in the case of different solid dielectrics, gases and polarity of the applied HV impulses will be presented and discussed in Section IV.

\section{BREAKDOWN CHARACTERISTICS}

\section{A. Breakdown voltage and time to breakdown}

Three fresh and cleaned dielectric samples of each material were stressed with $30 \mathrm{HV}$ impulses in each specific combination of the experimental parameters: each type of gas and solid material, and positive/negative impulse polarity.

The mean values of the breakdown voltage and time to breakdown were calculated using 90 experimental values of $V_{b r}$ and $t_{b r}$ ( 3 fresh samples $\times 30 \mathrm{HV}$ impulses). The corresponding values of the standard deviation in the breakdown voltage and time to breakdown, $V_{b r}$ and $t_{b r}$, were also calculated.

Figs. 9 and 10 show the mean values of $V_{b r}$ and $t_{b r}$ obtained under positive energisation, for each solid material in each gas. The error bars show the corresponding standard deviation values. As reference values, the breakdown voltage and time to breakdown obtained for 'No spacer' (NS) tests, with no solid dielectric placed between the electrodes, are also included in these graphs, for each gas type. It can be seen that, as compared with the 'No spacer' results, the introduction of the solid spacers into the test cell resulted in a statistically significant reduction in, both, the breakdown voltage, $V_{b r}$, and the time to breakdown, $t_{b r}$, except for the case of Delrin in a $\mathrm{CO}_{2}$ atmosphere. Delrin demonstrated the lowest reduction in the time to breakdown and breakdown voltage amongst all tested solid dielectric materials, and although $t_{b r}$ and $V_{b r}$ for Delrin in a $\mathrm{CO}_{2}$ atmosphere demonstrated a $\sim 20 \%$ and $\sim 3 \%$ reduction respectively as compared with the corresponding "no spacer" results, the error bars for these parameters which represent their standard deviation values overlap.

Figs. 9 and 10 also show that the most significant reduction in the values of $V_{b r}$ and $t_{b r}$ were obtained for all tested solid dielectric materials in a $\mathrm{N}_{2}$ atmosphere, while the lowest reduction in these values was obtained in a $\mathrm{CO}_{2}$ atmosphere.

Nevertheless, there is a statistically significant difference between the values of $V_{b r}$ and $t_{b r}$ obtained for different gasses for each of the solid spacer materials. The values of $V_{b r}$ are higher and $t_{b r}$ is longer for breakdown events in a $\mathrm{CO}_{2}$ atmosphere, as compared with the breakdown events in air and in $\mathrm{N}_{2}$. It was found that $V_{b r}$ and $t_{b r}$ were similar in the case of breakdown events in air and $\mathrm{N}_{2}$ : no statistically significant difference was observed for the breakdown parameters in these two gases, except for with the Macor ceramic sample where dry air had larger values of $V_{b r}$ and $t_{b r}$ compared to $\mathrm{N}_{2}$.

The standard deviation in $t_{b r}$ for $\mathrm{CO}_{2}$ was higher than that for the other two gases. This is due to the higher breakdown voltage obtained in a $\mathrm{CO}_{2}$ atmosphere: all breakdown events occurred on the peak plateau of the voltage waveform, resulting in a wider variation in $t_{b r}$, but not in the registered breakdown voltage.

Figs. 9 and 10 show that, in the case of the positive HV impulses, the variation in the relative permittivity of the spacers used in these tests is not translated into a statistically significant variation in the breakdown parameters, $V_{b r}$ and $t_{b r}$.

Contrary to the case of positive energisation, it was found that, when negative HV impulses were applied, both breakdown parameters, $V_{b r}$ and $t_{b r}$, obtained with the solid spacers located in the test system were statistically similar to, or greater than, the values of $V_{b r}$ and $t_{b r}$ obtained in the gas atmosphere only ('no spacer' breakdown events), as evident in Figs. 11 and 12.

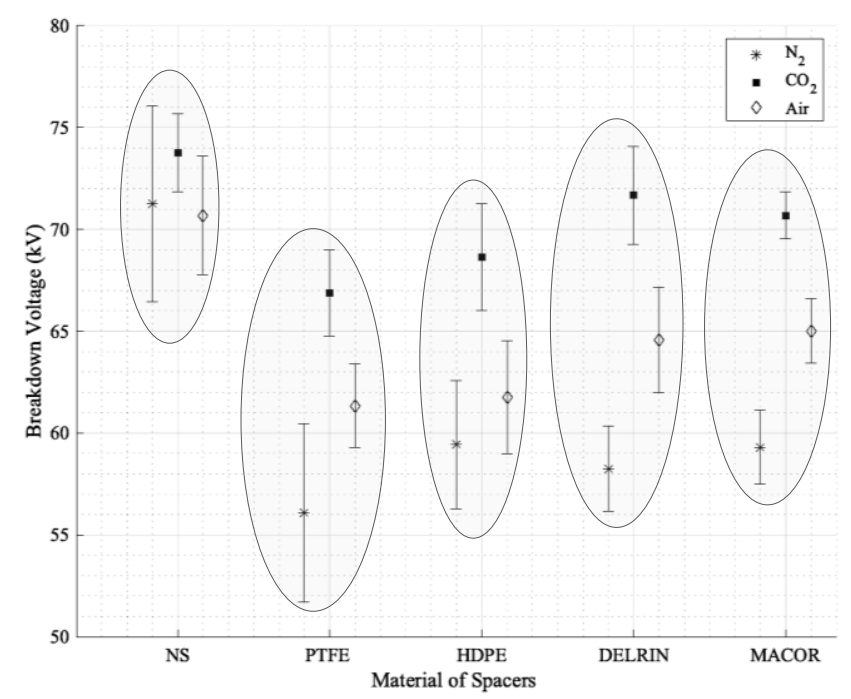

Fig. 9. Average values of the breakdown voltage, $V_{b r}$, for all tested gases and solid dielectrics, under positive energisation. NS - 'no spacer' breakdowns in gas. Error bars show the standard deviation in $V_{b r}$. 


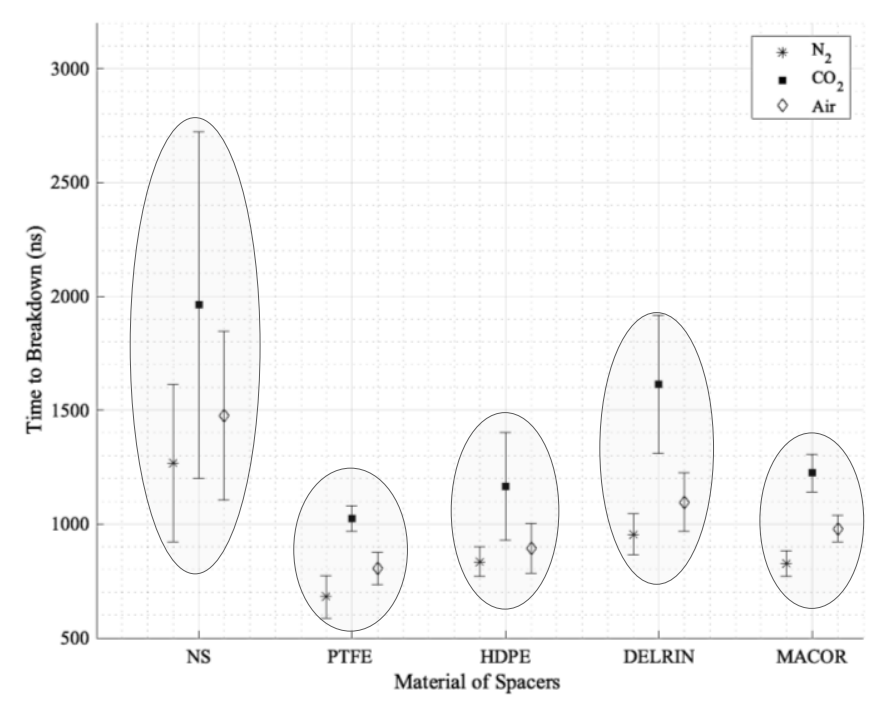

Fig. 10. Average values of the time to breakdown, $t_{b r}$, for all tested gases and solid dielectrics, under positive energisation. NS - 'no spacer' breakdowns in gas. Error bars show the standard deviation in $t_{b r}$

A statistically significant increase in both, $V_{b r}$ and $t_{b r}$, was observed for the breakdown events with a solid as compared with the breakdown parameters measured in air and $\mathrm{N}_{2}$ atmospheres. However, there was no statistically significant increase in the breakdown voltage with the inclusion of solid spacers in a $\mathrm{CO}_{2}$ atmosphere, compared to the breakdown voltage in this gas alone.

In the case of breakdown in a $\mathrm{N}_{2}$ atmosphere, a statistically significant increase in the negative breakdown voltage was obtained for all solid materials: $V_{b r}$ in $\mathrm{N}_{2}$ alone was $\sim 82 \mathrm{kV}$, however when PTFE, HDPE and Delrin spacers were included, $V_{b r}$ increased up to $\sim 97 \mathrm{kV}, \sim 99 \mathrm{kV}$ and $\sim 95 \mathrm{kV}$, respectively, as can be seen in Fig. 11. In the case of the Macor sample, it was found that $V_{b r} \approx 90 \mathrm{kV}$ in a $\mathrm{N}_{2}$ atmosphere.

In the case of dry air, an apparent increase in $V_{b r}$ was evident with an introduction of the solid dielectrics into the test system, however, the error bars for $V_{b r}$ obtained for different spacers overlap, Fig.11. This is a result of breakdown events taking place at the peak plateau of the voltage waveform, where the rate of voltage change is significantly lower than that during the front of the HVT impulse. Thus, a more accurate analysis of the breakdown behavior could be conducted based on $t_{b r}$, rather than on $V_{b r}$ in this particular case. Indeed, Fig. 12 demonstrates

that, in the case of dry air, a statistically significant increase in $t_{b r}$ was obtained for all solid dielectrics as compared with 'no spacer' breakdown events in air: $t_{b r}$ increases from $\sim 1.5 \mu \mathrm{s}$ to $\sim 2.8 \mu \mathrm{s}$ for PTFE, to $\sim 2.2 \mu \mathrm{s}$ for HDPE, to $\sim 2.5 \mu \mathrm{s}$ for Delrin, and to $\sim 2.9 \mu$ s for Macor, Fig. 12.

A similar, statistically significant, difference in $t_{b r}$ for solid spacers was obtained for the breakdown events registered in a $\mathrm{N}_{2}$ environment, with $t_{b r}$ increasing from $\sim 0.8 \mu \mathrm{s}$ ('no spacer') to $\sim 1.7 \mu$ s for PTFE, to $\sim 1.4 \mu$ s for HDPE, to $\sim 1.2 \mu$ s for Delrin, and to $\sim 1.7 \mu$ s for Macor, Fig. 12 .

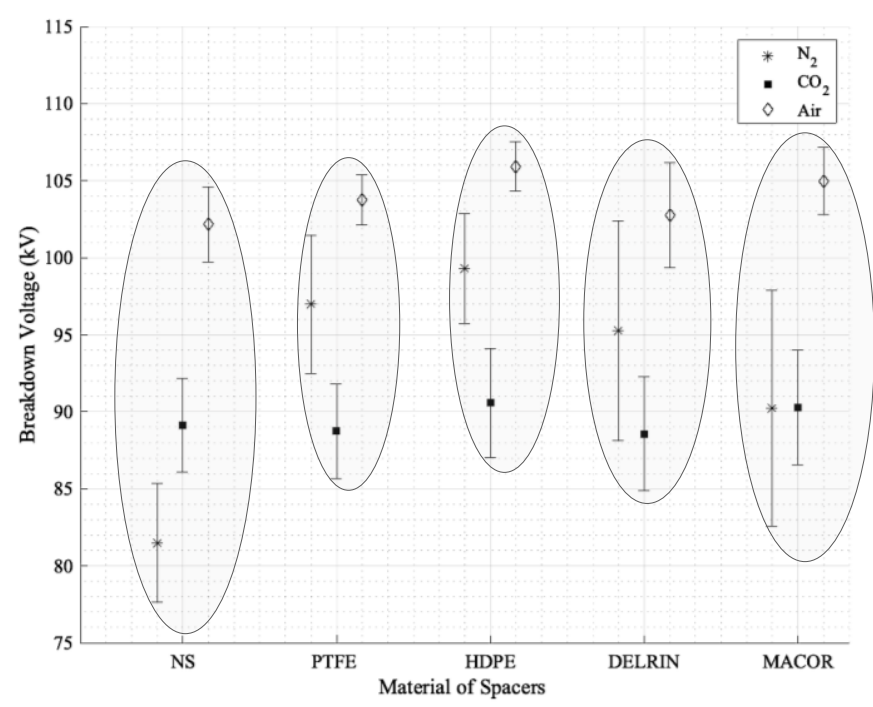

Fig. 11. Average values of the breakdown voltage, $V_{b r}$, for all tested gases and solid dielectrics, under negative energization. NS - 'no spacer' breakdowns in gas. Error bars show the standard deviation in $V_{b r}$.

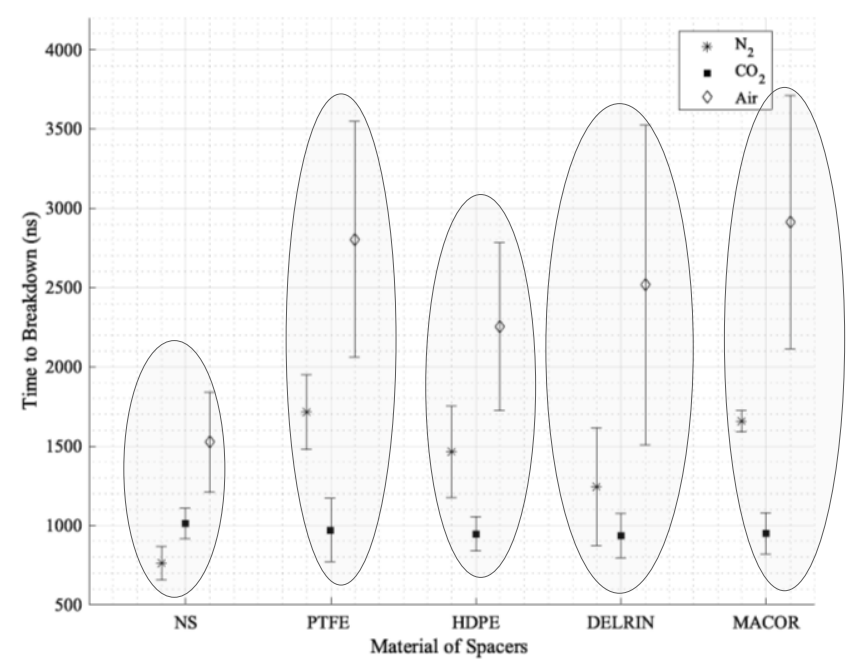

Fig. 12. Average values of the time to breakdown, $t_{b r}$, for all tested gases and solid dielectrics, under negative energization. NS - 'no spacer' breakdowns in gas. Error bars show the standard deviation in $t_{b r}$.

No statistically significant difference in $t_{b r}$ was found for 'no spacer' breakdown events in $\mathrm{CO}_{2}$ (gas only), and for breakdown events with the inclusion of spacers in a $\mathrm{CO}_{2}$ atmosphere. Also, the breakdown parameters obtained for negative HV impulses showed that there was no statistically significant difference in either, $V_{b r}$ or $t_{b r}$, for all tested solid materials. Thus, as in the case of the positive energisation, it can be stated that the relative permittivity of the solid spacers used in the present study does not significantly affect the flashover breakdown parameters in the present experimental conditions. This result can be explained by considering a potential initiation point of the prebreakdown streamer(s) at the triple junction, Fig. 3. As discussed in [10], for inter-electrode distances shorter than $\sim 5$ $\mu \mathrm{m}$, the probability of ionising collisions decreases, and the breakdown mechanism in such conditions is not based on the Townsend avalanche multiplication process. Therefore, it could 
be expected that the pre-breakdown streamer(s) will be initiated in $\sim 5 \mu \mathrm{m}$ or wider gaps. According to Fig. 5, at $\ell \sim(5-6) \mu \mathrm{m}$, the difference in the electric field magnitude on the surface of the $\mathrm{HV}$ electrode, $E_{e l}$, for different solid dielectric materials is $\sim 10 \%$. Thus, at such $\ell$, it can be stated that $\varepsilon_{r}$ does not significantly affect $E_{e l}$. This could be a potential reason for the fact that a statistically significant difference in $V_{b r}$ for the solid materials used in this study was not observed.

It also should be noted that there was no noticeable influence of the residual chemical species generated by initial spark discharge(s) on the parameters of the following breakdown events. The breakdown voltage and time to breakdown in each series of tests (i.e. for the same dielectric sample and gas in the test cell) did not demonstrate any decreasing or increasing tendencies which allowed for their statistical analysis through calculation of their mean values and standard deviation values. Moreover, the statistical analysis of the breakdown voltages and times to breakdown of 3 first breakdown events for each type of dielectric material (each new dielectric sample in the test cell filled with a fresh portion of gas was exposed to a HV impulse) demonstrated that there is no statistically significant difference between the mean values of $V_{b r}$ and $t_{b r}$ obtained using a dataset of the first breakdown events and the mean values of these parameters obtained using a complete dataset of 90 breakdown events. It could be explained by a relatively short life time of the charged species produced by the spark discharges (while the time interval between each impulse in the present tests was approximately $5 \mathrm{~min}$ ). For example, it was shown in [13] that the electron density demonstrates a 3-log reduction during a period of only 5-20 $\mu$ s after a spark discharge in air, i.e. $99.9 \%$ of electrons produced by the spark discharge plasma vanish in 10 's $\mu$ s after the discharge. In [14] it was shown analytically that the densities of the main ionic species produced by plasma discharges in air $\left(\mathrm{NO}^{+}, \mathrm{H}_{3} \mathrm{O}^{+}, \mathrm{NO}_{2}^{-}\right)$also demonstrate a to 4 $\log$ reduction after only 0.1-10 $\mu$ s. It also known that the typical recovery time of un-flashed, gas-filled plasma closing switches is in the sub-second time interval, [15].

\section{B. Breakdown path}

Open shutter photographs of all breakdown events were obtained, and the images of the breakdown channels were analysed and classified. It was observed that all breakdown channels initiated from the external edge of the HV electrode. This finding supports the statement that the enhanced electric field at the triple junctions, Fig.4, governs the breakdown initiation processes at the edge of the HV electrode. Also, no breakdown traces were observed at the upper surface of all tested cylindrical dielectric samples, this surface was in contact with the HV electrode, Fig.3. Therefore, it can be stated that no breakdown events were initiated at the edge of the locator pin in the hole of dielectric samples.

Eight different types of spark breakdown channels have been identified, based upon their propagation paths. Fig. 13 shows several examples of these breakdown channel geometries: (a) the entire breakdown path propagates through the bulk of the gas; (b) the breakdown path propagates across the solid-gas interface; (c) ) the breakdown propagates through the gas in the region close to the $\mathrm{HV}$ electrode and propagates along the insulator in the region close to the earth electrode; (d) the breakdown propagates attached to the surface of the dielectric in the region close to the HV electrode and through the gas in the region close to the earth electrode; (e) and (f) show examples of complex 'Gas-Surface-Gas discharge' and 'Surface-Gas-Surface' discharges, respectively; (g) and (h) show examples of a split spark channel, and of a multi-channel breakdown event, respectively.

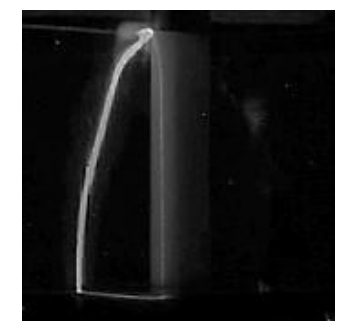

(a) Gas discharge, G-D

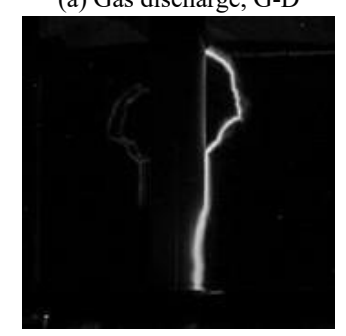

(c) Gas-Surface discharge, GS-D

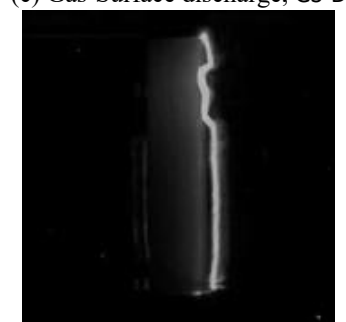

(e) Gas-Surface-Gas discharge, GSG-D

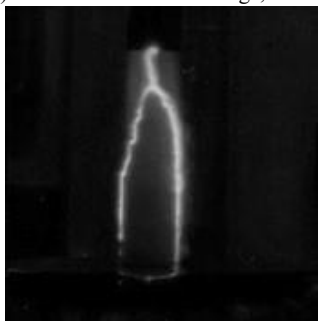

(g) Split discharge, SPL-D
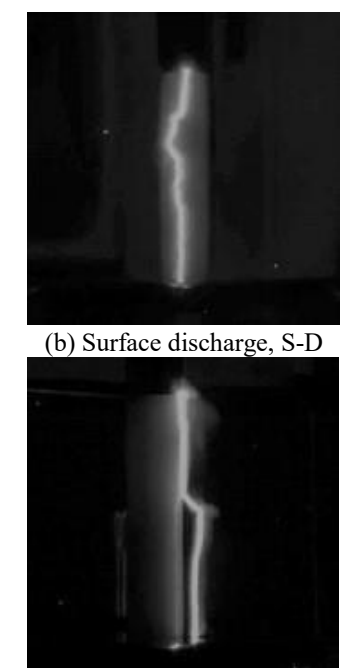

(d) Surface-Gas discharge, SG-D

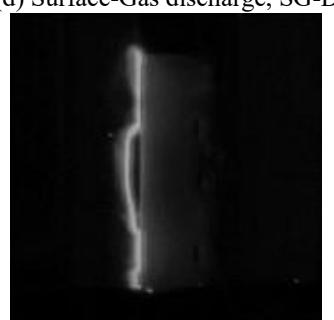

(f) Surface-Gas-Surface discharge, SGS-D

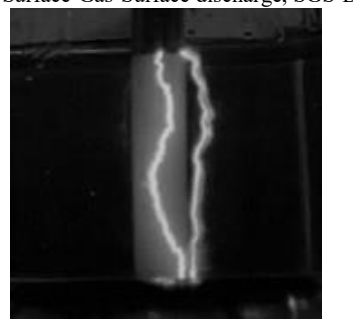

(h) Multi-channel discharge, MC-D
Fig. 13. Types of thermalized breakdown paths observed.

It should be noted that the breakdown events in the case of PTFE samples in $\mathrm{N}_{2}$ and air atmospheres behaved differently from those associated with the other solid materials in the same gases, due to a significant degree of carbonisation observed on the surface of PTFE samples after a few initial discharges, Fig. 14. It was noted that practically all discharges followed the same paths across the PTFE/ $\mathrm{N}_{2}$ interface, along the established carbonised paths. This phenomenon was observed in the case of $\mathrm{N}_{2}$ and air atmospheres, significantly less visible carbonisation was registered in the case of breakdown events in $\mathrm{CO}_{2}$. Thus, the classification of the breakdown channel paths in 
the cases of PTFE samples in $\mathrm{N}_{2}$ in particular is different from that of the three other solid dielectric materials in the same gases, where no such pre-determined breakdown paths were observed. For example, more than $96 \%$ of discharges propagated along the PTFE/ $\mathrm{N}_{2}$ interface (the S-D type in Fig.16), resulting in such cumulative, clearly visible carbon traces on the surface of PTFE samples. However, only $\sim 2 \%$ of discharges in the case of $\mathrm{HDPE} / \mathrm{N}_{2}$ interfaces propagated along the solid-gas interface.

However, it should be noted that carbonisation observed on the surface of PTFE samples did not affect the breakdown properties of the PTFE-gas interfaces. It was shown that there is no statistically significant difference between the mean values of the breakdown voltages or time to breakdown, $V_{b r}$ and $t_{b r}$, obtained using a dataset of the first breakdown events (there was no carbon traces on the surface of new solid dielectric samples) and the mean values of these parameters obtained using a dataset of all 90 breakdown events. Table II shows $V_{b r}$ and $t_{b r}$ obtained using these two datasets for PTFE in all tested gases.

TABLE II

$V_{B R}$ AND $t_{b r}$ FOR PTFE / GAS INTERFACES

\begin{tabular}{ccccc}
\hline \hline $\begin{array}{c}\text { Gas } \\
\text { Parameter }\end{array}$ & $\begin{array}{c}\text { First } \\
\text { breakdown } \\
\text { events, Pos. }\end{array}$ & $\begin{array}{c}90 \\
\text { breakdown } \\
\text { events, Pos. }\end{array}$ & $\begin{array}{c}\text { First } \\
\text { breakdown } \\
\text { events, Neg. }\end{array}$ & $\begin{array}{c}90 \\
\text { breakdown } \\
\text { events, Neg. }\end{array}$ \\
\hline $\begin{array}{c}\text { Air } \\
V_{b r}, \mathrm{kV}\end{array}$ & $62.59 \pm 3.70$ & $61.33 \pm 2.06$ & $\begin{array}{c}103.37 \pm \\
0.75\end{array}$ & $103.76 \pm 1.63$ \\
$\begin{array}{c}\text { Air } \\
t_{b r}, \mu \mathrm{s}\end{array}$ & $0.85 \pm 0.10$ & $0.81 \pm 0.07$ & $2.64 \pm 0.94$ & $2.81 \pm 0.74$ \\
$\mathrm{~N}_{2}$ & & & & \\
$V_{b r}, \mathrm{kV}$ & $58.91 \pm 3.33$ & $56.08 \pm 4.36$ & $99.63 \pm 3.50$ & $97.68 \pm 2.12$ \\
$\mathrm{~N}_{2}$ & & & & \\
$t_{b r}, \mu \mathrm{s}$ & $0.73 \pm 0.16$ & $0.680 \pm 0.09$ & $1.82 \pm 0.65$ & $1.71 \pm 0.24$ \\
$\mathrm{CO}_{2}$ & & & & \\
$V_{b r}, \mathrm{kV}$ & $67.90 \pm 1.71$ & $66.8 \pm 2.10$ & $86.29 \pm 1.35$ & $88.74 \pm 3.07$ \\
$\mathrm{CO}_{2}$ & & & & \\
$t_{b r}, \mu \mathrm{s}$ & $1.00 \pm 0.02$ & $1.02 \pm 0.06$ & $1.08 \pm 0.11$ & $0.97 \pm 0.20$ \\
& & & & \\
\hline \hline
\end{tabular}

The probability of each type of spark breakdown path was obtained by dividing the number of breakdown channels in each category by the total number of breakdown events. Figs. 15 - 20 present the results of an analysis of the breakdown paths observed for each solid material in each gas.

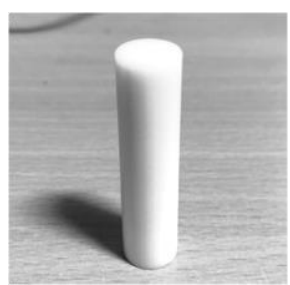

(a)

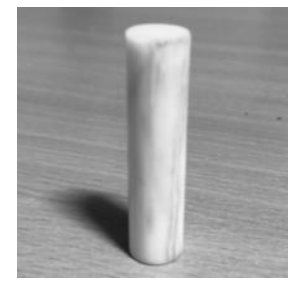

(b)
Fig. 14. PTFE sample used in $\mathrm{N}_{2}$ tests under negative energization. (a) fresh sample; (b) sample after 30 negative breakdowns.
Figs. 15 and 16 present the breakdown path classification for the breakdown events in $\mathrm{N}_{2}$ for positive and negative $\mathrm{HV}$ impulses, respectively, for HDPE, Delrin, PTFE, and Macor solid samples.

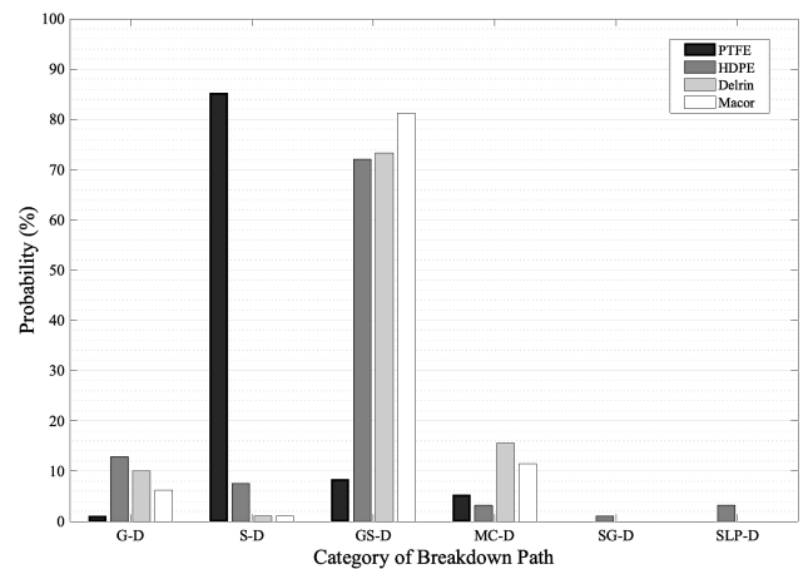

Fig. 15. Statistical distribution of the breakdown paths for all spacers in $\mathrm{N}_{2}$ under positive energisation.

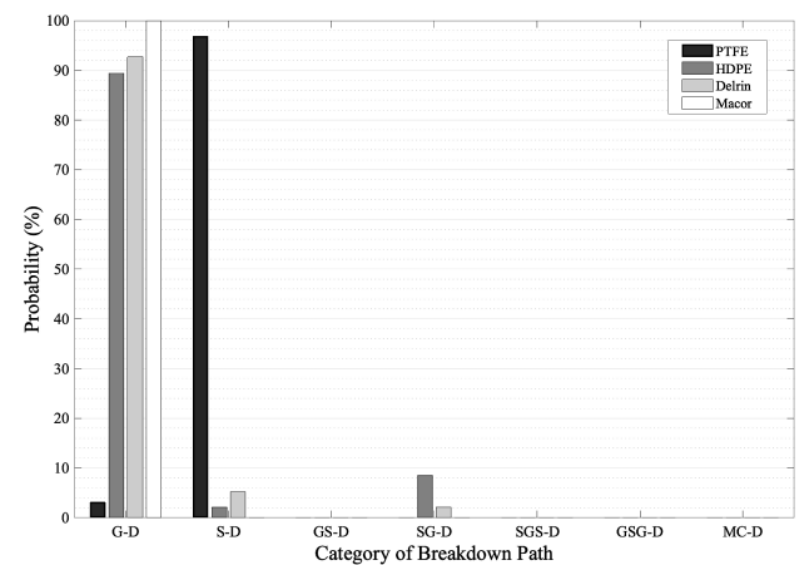

Fig. 16. Statistical distribution of the breakdown paths for all spacers in $\mathrm{N}_{2}$ under negative energisation.

It can be seen that, in the case of positive energisation for all solid dielectrics (except for PTFE, as discussed above), the most probable path of the breakdown spark channels is the "Gas-Surface" path, the GS-D type. However, in the case of negative energisation, the most probable path of the spark channel for HDPE, Delrin, and Macor samples is a discharge through the bulk of $\mathrm{N}_{2}$, the G-D type. For the PTFE samples, as discussed above, all discharges propagate along the PTFE-gas interface (the S-D type).

Figs. 17 and 18 show the statistical distribution of the discharge types obtained in the case of breakdown events in a $\mathrm{CO}_{2}$ atmosphere. 


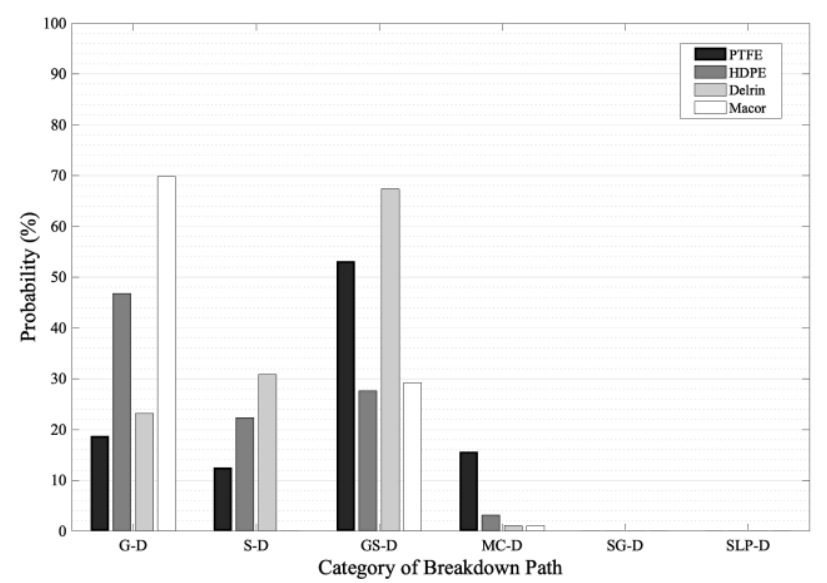

Fig. 17. Statistical distribution of the breakdown paths for all spacers in $\mathrm{CO}_{2}$ under positive energisation.

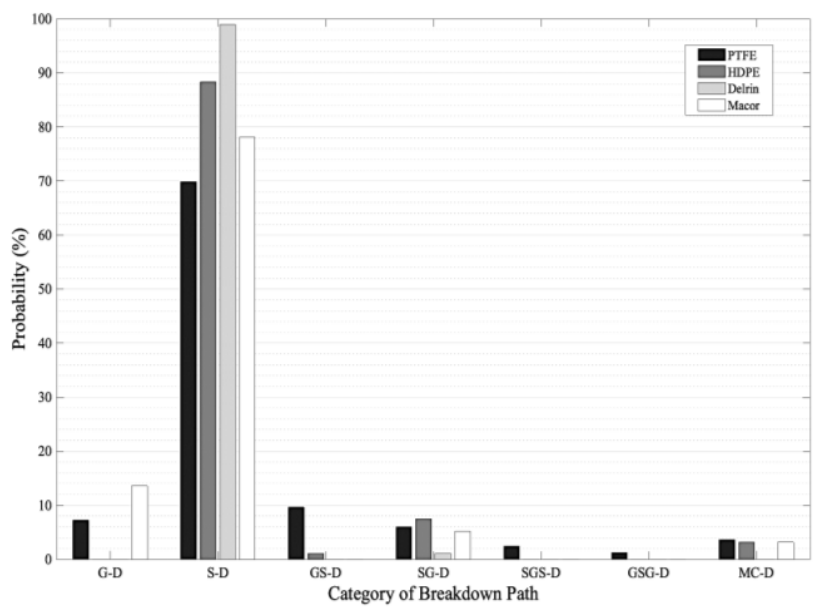

Fig. 18. Statistical distribution of the breakdown paths for all spacers in $\mathrm{CO}_{2}$ under negative energisation.

It can be seen that, in the case of positive energisation, the breakdown channel types are distributed between the G-D, S-D and GS-D types, Fig. 17. With the Macor ceramic spacer, $70 \%$ of channels observed were of the G-D type, and $\sim 30 \%$ were GSD spark channel paths, while Delrin showed the opposite tendency: $\sim 22 \%$ discharges of G-D type, and $\sim 68 \%$ of GS-D type. Under negative energisation in a $\mathrm{CO}_{2}$ atmosphere (Fig. 18), most of the spark channels, for all solid dielectrics, solid-gas flashover spark breakdown channels, the S-D type.

Figs. 19 and 20 show the statistical distribution of the discharge types obtained in the case of dry air. As in the case of positive energisation in a $\mathrm{CO}_{2}$ atmosphere, the breakdown channel topologies in air are distributed between the G-D, S-D and GS-D types, Fig. 19. Delrin demonstrated $\sim 65 \%$ of GasSolid discharges (the GS-D type), and $\sim 12 \%$ discharges in both, G-D and S-D, types. PTFE, HDPE and Macor demonstrated $30 \%-42 \%$ of the GS-D type of discharges. However, in the case of negative energisation (Fig. 20), the most probable breakdown channel type, for all solid materials, was the G-D category, i.e. spark discharges that propagate through the bulk of the gas (dry air).

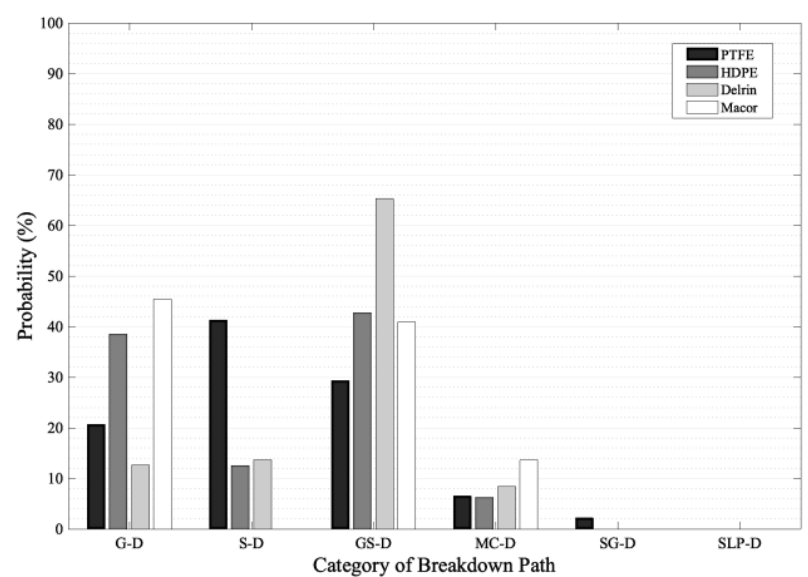

Fig. 19. Statistical distribution of the breakdown paths for all spacers in air under positive energisation.

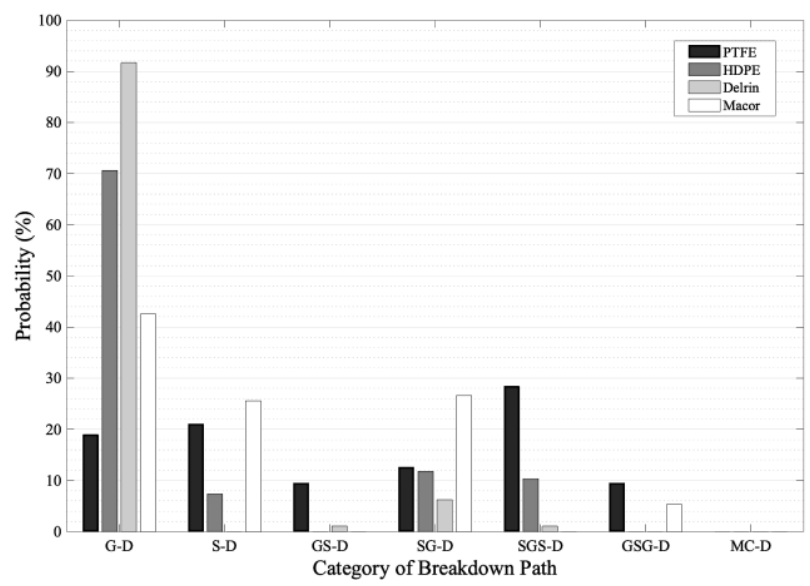

Fig. 20. Statistical distribution of the breakdown paths for all spacers in air under negative energisation.

In general, it was found that the probability of the "GasSurface-Gas" discharges (GSG-D) and the "Surface-GasSurface" discharges (SGS-D) was lower as compared with the probabilities of the G-D, S-D and GS-D discharge types, for all tested combinations of dielectric/gas/polarity.

Multi-channel (MC-D) and split (SPL-D) discharges were observed in the $\mathrm{CO}_{2}$ atmosphere under negative energisation in less than $5 \%$ of cases. For positive energisation, a higher probability of multi-channeling was obtained - this probability was in the range from $\sim 3 \%$ up to $\sim 15 \%$, depending upon the solid dielectric material. The Macor ceramic spacers demonstrated a notable number of multi-channel discharge events in both, $\mathrm{N}_{2}$ and air.

\section{DISCUSSION AND CONCLUSIONS}

The obtained results demonstrate that the introduction of solid dielectric spacers between the HV and ground electrode can significantly change the breakdown characteristics of the gas-filled HV system. The functional breakdown behaviour and the degree of these changes depend upon the polarity of the applied HV impulses, and the gas type used in the breakdown tests. However, for the same gas type and impulse polarity no statistically significant changes in the breakdown parameters, 
$V_{b r}$ and $t_{b r}$, were established between the different solid dielectrics used in this study with an exception of PTFE stressed with positive impulses, in this case the error bars for $V_{b r}$ and $t_{b r}$ are close but don't overlap with the error bars for the same parameters obtained for Delrin and Macor.

Potentially, this experimental result can be supported by the analysis of the field magnitude, $E_{e l}$, on the electrode surface at the triple junctions, Fig.5. As it was discussed in Section II(E), a higher value of $\varepsilon_{r}$ of the solid dielectric will not necessarily result in the largest value of $E_{e l}$. Therefore, the breakdown voltage obtained in present topologies may not decrease with an increase in the relative permittivity of the solid spacer, as was reported in [16].

\section{A. Negative impulses}

It was found that, in the case of negative HV impulses, both parameters, $V_{b r}$ and $t_{b r}$, increased when a solid spacer, of any material, was included between the HV and ground electrodes, in both air and $\mathrm{N}_{2}$ atmospheres (as compared with 'no spacer' values). However, in a $\mathrm{CO}_{2}$ atmosphere, the introduction of the solid insulators did not result in statistically significant changes in $V_{b r}$ and $t_{b r}$, as compared with breakdown events in the bulk of this gas ('no spacer' tests).

A comprehensive analysis of the open shutter photographs of the spark channels at breakdown demonstrated that in both, $\mathrm{N}_{2}$ and air, atmospheres, the majority of the breakdown spark channels propagated through the bulk of these gases (G-D type). However, in the case of $\mathrm{CO}_{2}$, the majority of the breakdown channels propagated across the solid-gas interfaces (S-D type). It may be suggested that, a longer path of the breakdown channel in the case of the S-D discharge type resulted in a longer time to breakdown (and a higher breakdown voltage).

The reason for the spark breakdown channels to attach to the solid surface in the case of $\mathrm{CO}_{2}$ atmospheres, and to propagate through the bulk of the gas in the case of both, $\mathrm{N}_{2}$ and air, atmospheres is not fully understood, and requires further investigation. Potentially, incomplete negative pre-breakdown streamers which develop across the solid-air or solid- $\mathrm{N}_{2}$ interfaces simultaneously with the steamer(s) through the bulk of the gases, [17], can lead to the formation of a negative residual surface charge, which can stimulate subsequent negative streamers to propagate through the gas, rather than across the solid-gas interface.

\section{B. Positive impulses}

In the case of positive energisation, the introduction of solid spacers into the test system resulted in statistically significant reductions in $V_{b r}$ and $t_{b r}$ compared to those obtained for 'no spacer' breakdowns in gas. This reduction was registered for all gases and solid dielectrics stressed with positive HV impulses. The analysis of the captured open shutter images demonstrated that the majority of the breakdown channels belonged to the "Gas-Surface Discharge" and "Gas Discharge" types. Thus, it may be argued that the development of the positive streamer(s) across the solid-gas interface is a faster process than the process of the development of positive streamer(s) though the bulk of the gas, in the absence of any solid dielectric spacer. The role of the positive surface charge which can be developed by photons emitted by the positive streamer heads could be significant, and this surface charge may help to accelerate those positive streamers which propagate in the vicinity of the solidgas interface, due to the emission of secondary electrons from the dielectric surface. For example, in [18] it was found that both, photoemission and secondary electron emission, are major sources of electrons emitted from the solid-gas interface. Both mechanisms can trigger the emission of electrons from the solid-gas interface, and these secondary electrons could promote the development of streamer(s) across/in the vicinity of the interface. However, this hypothesis and other potential factors which may affect the development of positive streamers across solid-gas interfaces require further investigation.

The obtained results will help in the analysis of the basic mechanisms responsible for the breakdown processes in different solid-gas insulation systems stressed with $\mathrm{HV}$ impulses. These results can be used in practical engineering applications, which require detailed information on the breakdown/flashover characteristics of solid spacers in different gases. Thus, the information obtained in this study will help in optimisation and coordination of electrical insulation in gas-filled insulating systems used in high voltage and pulsed power systems.

\section{REFERENCES}

[1] S. J. Dale, "Future development in solid dielectric insulators for compressed gas insulated transmission systems," in Conference on Electrical Insulation \& Dielectric Phenomena - Annual Report 1981, 2628 Oct. 1981 1981, pp. 404-409.

[2] T. S. Sudarshan and R. A. Dougal, "Mechanisms of Surface Flashover Along Solid Dielectrics in Compressed Gases: a Review," IEEE Transactions on Electrical Insulation, vol. EI-21, no. 5, pp. 727-746, 1986.

[3] J. G. Trump and J. Andrias, "High-Voltage D-C Flashover of Solid Insulators in Compressed Nitrogen," Transactions of the American Institute of Electrical Engineers, vol. 60, no. 11, pp. 986-990, 1941, doi: 10.1109/T-AIEE.1941.5058218.

[4] L. Lazaridis and P. Mikropoulos, "Flashover along cylindrical insulating surfaces in a non-uniform field under positive switching impulse voltages," IEEE Transactions on Dielectrics and Electrical Insulation, vol. 15, pp. 694-700, 07/01 2008.

[5] C. Tran Duy et al., "Partial discharges at a triple junction metal/solid insulator/gas and simulation of inception voltage," Journal of Electrostatics, vol. 66, no. 5, pp. 319-327, 2008/05/01/ 2008.

[6] L. Trémas, O. Lesaint, N. Bonifaci, and F. Gentils, Breakdown in air along insulating solid surfaces of different natures, parallel or perpendicular to the field direction. 2016, pp. 412-415.

[7] J. T. Krile, A. A. Neuber, J. C. Dickens, and H. G. Krompholz, "DC flashover of a dielectric surface in atmospheric conditions," IEEE Transactions on Plasma Science, vol. 32, no. 5, pp. 1828-1834, 2004.

[8] A. Peschot, N. Bonifaci, O. Lesaint, C. Valadares, and C. Poulain, "Deviations from the Paschen's law at short gap distances from $100 \mathrm{~nm}$ to $10 \mu \mathrm{m}$ in air and nitrogen," Applied Physics Letters, vol. 105, no. 12, p. 123109, 2014.

[9] L. Trémas, O. Lesaint, N. Bonifaci, B. Ohl, and F. Gentils, "Influence of the solid material nature on the inception of creeping discharges in air," in 2017 IEEE Conference on Electrical Insulation and Dielectric Phenomenon (CEIDP), 22-25 Oct. 2017 2017, pp. 548-551.

[10]R. E. Jorgenson, L. K. Warne, A. A. Neuber, J. Krile, J. Dickens, and H. G. Krompholz, "Effect of dielectric photoemission on surface breakdown: An LDRD report," Sandia Report SAND2000-3044, 2003.

[11] G.L.Weissler, Photoionization in gases and photoelectric emission from solids. In: Electron-Emission Gas Discharges I / Elektronen-Emission Gasentladungen I. Encyclopedia of Physics / Handbuch der Physik, vol 4 / 
21. Springer, Berlin, Heidelberg.(1956) https://doi.org/10.1007/978-3-64245844-6 4.

[12]M. Fujihira and H. Inokuchi, "Photoemission from polyethylene," Chemical Physics Letters, vol. 17, no. 4, pp. 554-556, 1972.

[13] J.Miles, C. Murray, A. Ross, K. Lemmer, J. Russell and S. Adams, "Time resolved electron density and temperature measurements via Thomson scattering in an atmospheric nanosecond pulsed discharge", Plasma Sources Science and Technology, vol. 29, n. 7, 07LT02 (5pp), 2020.

[14] Y. Sakiyama, D. B. Graves, "Efficient modeling of atmospheric pressure surface micro-discharge plasma chemistry", Plasma Sources Sci. Technol. 22 (2013) 012003 (5pp)

[15] G. J. J. Winands, Z. Liu, A. J. M. Pemen, E. J. M. van Heesch, and K. Yan "Long lifetime, triggered, spark-gap switch for repetitive pulsed power applications", Review Of Scientific Instruments, 76, 085107, 2005

[16] C. Gomes, V. Cooray, and M. Rahman, "Breakdown characteristics and optically visible discharge paths of surface flashover," in 2012 IEEE Conference on Sustainable Utilization and Development in Engineering and Technology, 6-9 Oct. 2012 2012, pp. 111-116.

[17]X. Meng, H. Mei, L. Wang, Z. Guan, and J. Zhou, "Characteristics of streamer propagation along insulation surface: influence of shed configuration," IEEE Transactions on Dielectrics and Electrical Insulation, vol. 23, no. 4, pp. 2145-2155, 2016.

[18] S. Mahajan and T. Sudarshan, "Measurement of electrical avalanches and optical radiation near solid insulators in high pressure (up to $0.3 \mathrm{MPa}$ ) nitrogen gas," Journal of applied physics, vol. 69, no. 5, pp. 2877-2884, 1991.

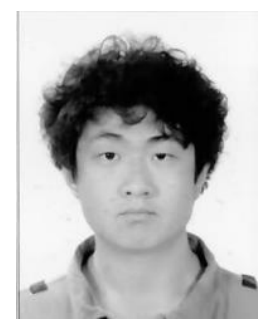

Zhe Wang received the B.Eng. and MSc. degrees from the University of Strathclyde, Glasgow, U.K., in 2016 and 2017.

Currently he is pursuing his Ph.D. degree in High Voltage Technology at the Department of Electronic and Electrical Engineering, University of Strathclyde, Glasgow, U.K. His current research interests include the application of surface discharge in pulse power system.

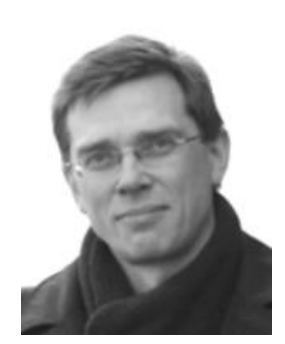

Igor V. Timoshkin (M'07-SM'14) received the degree in physics from Moscow State University, Moscow, Russia, in 1992, and the Ph.D. degree from the Imperial College of Science, Technology and Medicine (ICSTM), London, U.K., in 2001.

He was a Researcher at Moscow State Agro-Engineering University, Moscow, and then at the Institute for High Temperatures of Russian Academy of Sciences, Moscow. In 1997 he joined ICSTM. Then he joined the Department of Electronic and Electrical Engineering, University of Strathclyde, Glasgow, U.K., in 2001, where he became a Reader in 2016. His current research interests include pulsed power, transient spark discharges, environmental applications of non-thermal plasma discharges. Dr. Timoshkin is a Voting Member of the Pulsed Power Science and Technology Committee in the IEEE Nuclear and Plasma Science Society; a member of International Advisory Committee of the IEEE Conference on Dielectric Liquids and a member of the International Scientific Committee of the Gas Discharges and Their Application Conference.
Mark P. Wilson (M'10) was born in Stranraer, Scotland, in 1982. He received the B.Eng. (with honours), M.Phil., and $\mathrm{Ph} . \mathrm{D}$. degrees in electronic and electrical engineering from the University of Strathclyde, Glasgow, U.K., in 2004, 2007, and 2011, respectively. $\mathrm{He}$ is presently based in the High Voltage Technologies research group at the University of Strathclyde, where his research interests include interfacial surface flashover, nanodielectrics, and the practical applications of high power ultrasound, corona discharges, and pulsed electric fields. Mark is a member of the IEEE Nuclear and Plasma Sciences Society, from whom he received a Graduate Scholarship Award in 2011, the IEEE Dielectrics and Electrical Insulation Society, and the IET.

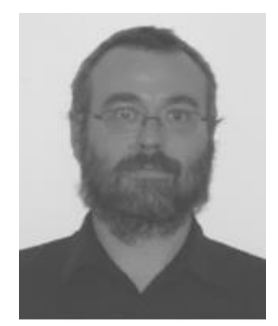

Martin J. Given (M'99-SM'11) received the B.Sc. degree in physics from the University of Sussex, Brighton, U.K., in 1981, and the Ph.D. degree in electronic and electrical engineering from the University of Strathclyde, Glasgow, U.K., in 1996.

He is currently a Senior Lecturer with the Department of Electronic and Electrical Engineering, University of Strathclyde. His current research interests include aging processes and condition monitoring in solid and liquid insulation systems, high-speed switching, and pulse power.

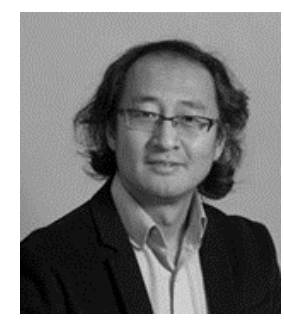

Tao Wang received the B.Eng and M.Sc degrees from Northeast China Dianli University (China) in 1993 and 1996 respectively, and the Ph.D. degree from the University of Strathclyde (Glasgow, UK) in 2005. He then joined the Newland Entech (China) as a research fellow developing high efficiency industrial ozone generator. He joined the department of Electronic and Electrical Engineering of University of Strathclyde as a lecturer in 2010. His research interests include non-thermal gas discharges and their applications in gas synthesis, water disinfection and advanced oxidation process in water.

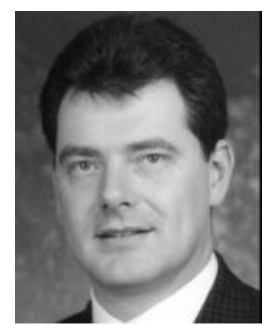

Scott J. MacGregor (M'95-SM'14) received the B.Sc. and Ph.D. degrees from the University of Strathclyde, Glasgow, U.K., in 1982 and 1986, respectively.

He was a Pulsed-Power Research Fellow in 1986 and a Lecturer in pulsed-power technology in 1989. In 1994, he became a Senior Lecturer, with a promotion to a Reader and a Professor of High Voltage Engineering, in 1999 and 2001, respectively. In 2006 and 2010, he became the Head of the Department of Electronic and Electrical Engineering and the Executive Dean of the Faculty of Engineering, and has been the Vice-Principal with the University of Strathclyde, since 2014. His current research interests include high-voltage 
pulse generation, high-frequency diagnostics, high-power repetitive switching, high-speed switching, electronic methods for food pasteurization and sterilization, the generation of high-power ultrasound (HPU), plasma channel drilling, pulsed-plasma cleaning of pipes, and the stimulation of oil wells with HPU. Prof. MacGregor was a recipient of the 2013 IEEE Peter Haas Award. He was an Associated Editor of the IEEE Transitions On Dielectrics and Electrical Insulation in 2015. 